\title{
Diseño y aplicación de un ciclo de innovación docente en Climatología
}

\section{Design and application of a teaching innovation cycle in Climatology}

MÓNICA AgUILAR-ALBA

ORCID: https://orcid.org/0000-0003-3386-3710

Universidad de Sevilla

Departamento de Geografía Física

y Análisis Geográfico Regional

malba@us.es

Fecha de recepción: 03-06-2019.

Fecha de aceptación: 10-06-2019.

DOI: http://dx.doi.org/10.12795/9788447221912.009

Pp.: 225-245 
Se presentan los resultados del diseño y aplicación de un ciclo de mejora en el aula en el bloque de Climatología de la asignatura Climatología y Biogeografia, obligatoria de segundo curso del Grado en Geografía y Gestión del Territorio. El objetivo es que los alumnos comprendan los factores explicativos zonales que determinan los principales tipos de climas del mundo. Este objetivo teórico comienza con el manejo de datos agrupados por bandas climáticas zonales, según la clasificación de W. Köppen. La aplicación del ciclo ha permitido constatar una realidad en los modelos y conocimientos previos de los estudiantes, que hace necesario cambiar el modelo de enseñanza-aprendizaje y los contenidos de la asignatura. Los resultados han conseguido mejorar de forma notable el rendimiento y las competencias de los estudiantes poniendo de manifiesto las dificultades y planteando nuevos retos.

Palabras clave: Geografía y gestión del territorio, climatología, docencia universitaria, experimentación docente universitaria, clasificaciones climáticas.

\section{Abstract}

The design and application of a Teaching Improvement Cycle are presented carried out in the climatology part of the second year course Climatology and Biogeography of the Degree in Geography and Territory Management. The main objective is for the students to understand the explanatory factors that determine the main climatic types in the world. This theoretical objective is approached from the management of data grouped by zonal climatic bands following the classification of W. Köppen. The application of the Teaching Improvement Cycle showed different student's models and previous knowledge than expected showing the need to change the teaching-learning model and the contents of the subject. The results have significantly improved the performance and skills of the students, highlighting the learning difficulties and posing new challenges.

Key words: Geography and territorial management, climatology, university teaching, university teaching experimentation, climatic classifications. 


\section{Contexto}

La asignatura propuesta para el ciclo de mejora en el aula (CIMA) es Climatología y Biogeografía, que se imparte como obligatoria en el segundo curso del Grado en Geografia y Gestión del Territorio y en el Doble Grado en Geografía y Gestión del Territorio e Historia. Incluye contenidos fundamentales de la geografia fisica que se han iniciado en la materia Geografia general fisica de primer curso. Tradicionalmente constituían asignaturas separadas, impartidas cada una en un cuatrimestre, hasta ser unificadas en el último plan de estudios, pasando a ser dos bloques temáticos. Esto plantea notables dificultades, tanto para alumnos como para profesores, en el aprendizaje de los conocimientos fundamentales en ambas temáticas por diversas razones: un programa denso y de contenidos muy diferentes, dos profesores -uno para cada bloque temático-, escaso tiempo (30 horas/bloque), etc.

Los ciclos de mejora propuestos en el marco del programa FIDOP de la Universidad de Sevilla se han desarrollado para el bloque de climatología del grupo de tarde, con 25 alumnos matriculados, de los que sólo asisten a clase unos 14, muchos de ellos repetidores, otros trabajando y dos estudiantes estadounidenses que realizan una estancia en el marco del programa de los cursos concertados que oferta la Facultad de Geografia e Historia. El horario de la asignatura, de 15.00 a 17.00 horas, unido a una aula estrecha, ruidosa y con bancas fijas alineadas hacia la mesa del profesor, suponen unas condiciones para la docencia poco gratas. El reparto temporal de los bloques en el cuatrimestre ha obligado a realizar el segundo CIMA antes de concluir las sesiones explicativas, lo cual ha llevado a cometer algunos errores que comentaremos en este artículo. No obstante, y a pesar de este contexto poco favorable, los alumnos han mostrado interés y, personalmente, la realización de los ciclos de mejora ha supuesto un aliciente para superar estas dificultades y una renovación de mi motivación docente. 


\section{Diseño previo del ciclo de mejora en el aula}

Los contenidos elegidos para el desarrollo de los ciclos de mejora son de carácter teórico-práctico y corresponden a dos temas dedicados al estudio de los diferentes climas de la tierra. El diseño de los dos ciclos de mejora se plantea sobre la pregunta: ¿Por qué existen tipos de climas diferentes en el planeta? Las actividades diseñadas pretenden que los alumnos comprendan mejor los factores explicativos que determinan los principales tipos de climas del mundo (climas regionales). El aprendizaje de las clasificaciones climáticas suele abordarse desde una perspectiva teórica y descriptiva que resulta dificil de asimilar. Por otro lado, los factores explicativos de los tipos de clima no suelen impartirse relacionándolos con los contenidos relativos a la radiación solar, balance de energía y circulación atmosférica. La intención es aprenderlos desde una perspectiva aplicada, partiendo de datos originales de climas del mundo, tantos como alumnos participen, agrupados por bandas climáticas zonales (macroclimas). Experiencias similares se han llevado a cabo como proyectos de innovación docente por parte de Rodríguez-Puebla en la Universidad de Salamanca (Rodríguez Puebla, 2010) y, en concreto, abordando las clasificaciones climáticas, como es el caso de Petre y colaboradores de la Universidad de Alcalá (Petre et al., 2014)Climatology and Air Pollution third year course of the Bachelor of Environmental Science at University of Alcalá.The collection of various forms of evidence of achievement of learning outcomes provides apart from its basic meaning (learning outcomes that allow students and others to assess the efforts, achievements, difficulties,strengths and weaknesses.

\section{Mapa de contenidos}

El mapa de contenidos parte de la pregunta ¿qué factores determinan el clima de un lugar? Los contenidos conceptuales que recoge están relacionados, por un lado, con la radiación solar incidente como factor principal que determina, según la latitud, el régimen de temperaturas. El 
segundo factor explicativo es la circulación general de la atmósfera y los centros de acción, responsables más importantes del régimen de precipitaciones. Ambos se pueden observar en un diagrama climático que muestra el régimen de precipitación y de temperatura. La descripción de las características del clima de un lugar debe buscar explicaciones fisicas (Rodríguez Puebla, 2010).

A pesar de su sencillez, la construcción de un climograma a partir de datos originales no es una tarea obvia para los estudiantes de segundo curso y se debe, fundamentalmente, a la necesidad del manejo, organización y estimación estadística de los registros hasta sintetizar correctamente las variables. Por esta razón se plantea la elaboración de este gráfico elemental como contenido procedimental que permite, al mismo tiempo, acercarse a las fuentes de información, Añade también contenidos actitudinales a través de la valoración, comprensión de la dificultad y compromiso de las instituciones en este proceso.

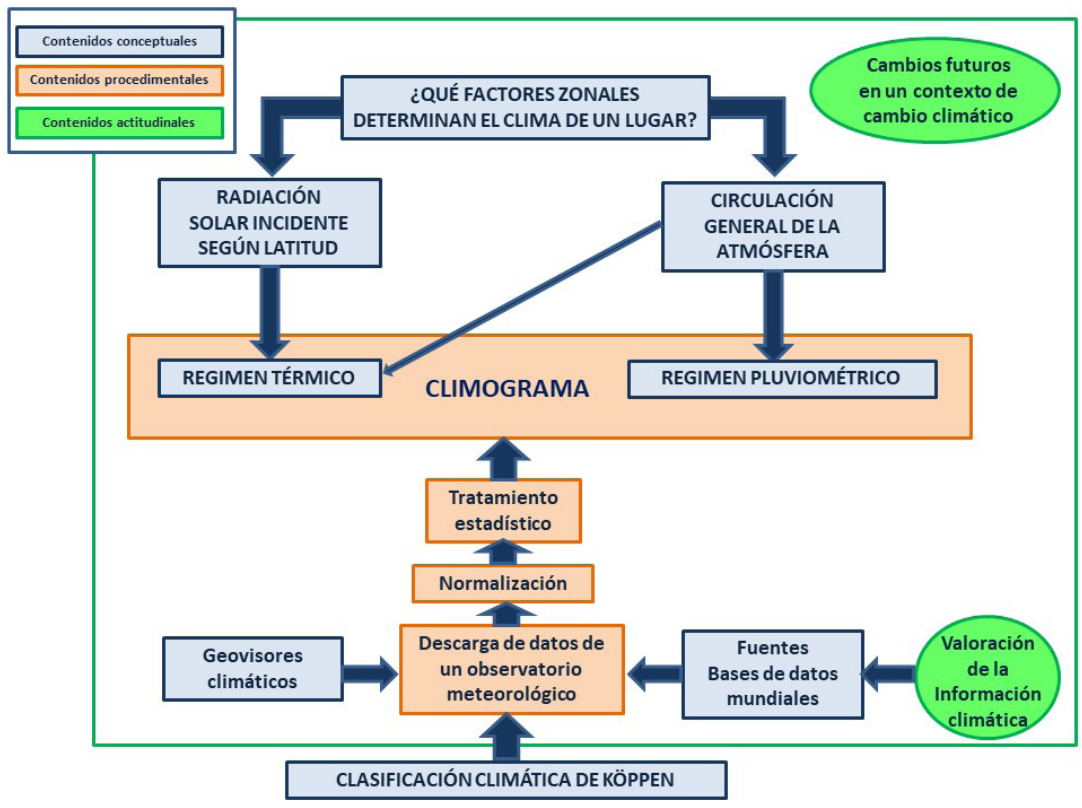

Figura 1: Mapa de contenidos para el ciclo de mejora docente

Jornadas de Formación e Innovación Docente del Profesorado | № 2 (2019) Esta obra se distribuye con la licencia Creative Commons Reconocimiento-NoComercial-SinObraDerivada 4.0 Internacional (CC BY-NC-ND 4.0.) 
Como se aprecia en la figura 1, los contenidos procedimentales parten de la clasificación climática mundial de W. Köppen, como base para seleccionar lugares del mundo con climas diferentes. La búsqueda de los datos se realiza a través de un visor climático (http://www.globalclimatemonitor.com) (Climate Research Group, s. f.). Este producto ha sido desarrollado por el Grupo de Climatología de la Universidad de Sevilla al que pertenece la autora (Camarillo-Naranjo, Álvarez-Francoso, Limones-Rodríguez, Pita-López, \& Aguilar-Alba, 2018). Los visualizadores de datos espaciales permiten disponer de información pública, libre, accesible y de calidad, objetivos de la ciencia actual y un reto aún para la climatología (Edwards, Mayernik, Batcheller, Bowker \& Borgman, 2011).

En el mapa de contenidos observamos una línea verde que enmarca casi la totalidad del mismo indicando su carácter global de contenidos actitudinales debido al contexto de cambio climático.

\section{Modelo metodológico posible y secuencia de actividades}

La actividad principal de los ciclos de mejora se centra en el trabajo en equipo con el cometido de buscar información y llegar a comprender qué factores zonales condicionan el régimen de temperaturas y precipitación de su macroclima (TG). Durante el desarrollo de la actividad la profesora participa resolviendo dudas, orientando y explicando las cuestiones que los alumnos demandan. Las conclusiones del grupo deben plasmarse en una presentación que van elaborando mientras trabajan. En casa pueden completarla según crean conveniente (TPC), matizando sobre el subtipo climático que cada uno tiene asignado dentro del grupo. 


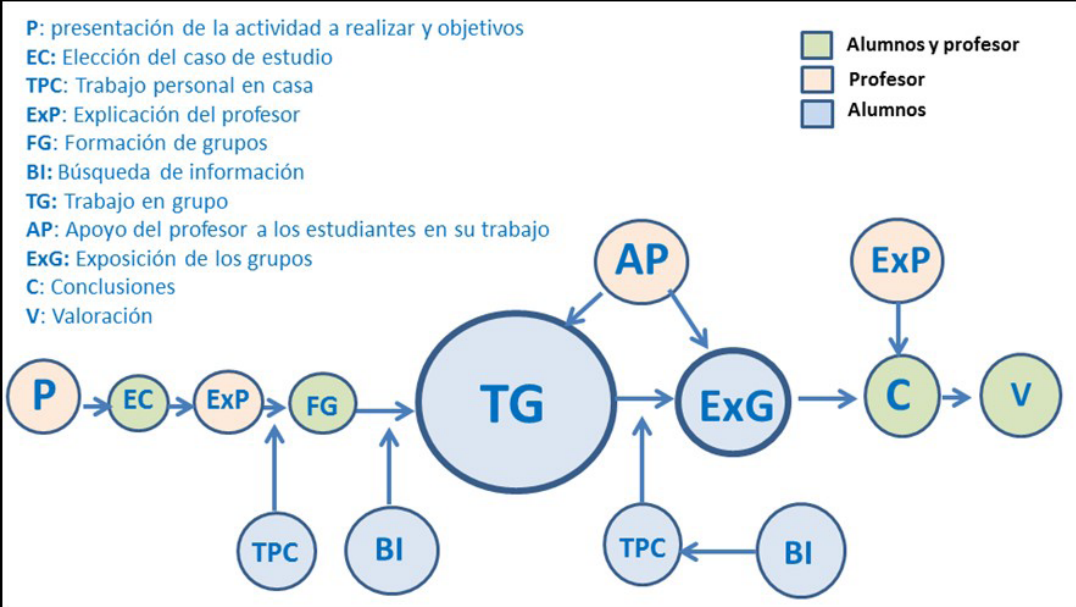

Figura 2: Modelo metodológico posible para ciclo de mejora en el aula

Como hemos comentado en el apartado anterior, las actividades de los ciclos de mejora pretenden que los alumnos partan de una pregunta-problema. Inicialmente los alumnos se dividen en grupos, formados a partir de la elección personal de un lugar perteneciente a los 5 macroclimas según la clasificación de W. Köppen (Climas A, B, C, D y E) y, posteriormente, seleccionan un subtipo diferente dentro de cada clase. De esta forma cada grupo trabaja colectivamente la caracterización general y la búsqueda de los factores explicativos del macroclima asignado (se forman por tanto 5 grupos en la clase). Una vez asignados los lugares, la profesora presenta el visor Global Climate Monitor como fuente de descarga los datos y explica el tratamiento de los datos hasta llegar a construir un climograma (ExP). Aprovechar las oportunidades que los nuevos datos y escenarios climáticos puedan presentar (Overpeck, Meehl, Bony, \& Easterling, 2011), mejora la preparación de los estudiantes en sus posibilidades laborales futuras. Cada estudiante descarga los datos de temperatura y precipitación de un lugar del mundo, elegido según sus preferencias, y los procesa hasta elaborar un (TPC). Se forman los grupos (FG) por macroclimas y se les pide que traigan 
manuales, libros de climatología y ordenadores para la sesión de trabajo en grupo (BI).

Finalmente, los resultados de las actividades en grupo y personales deben exponerse explicando los factores zonales que determinan los grandes tipos climáticos y los subtipos (ExG). La exposición sirve como actividad de contraste para evaluar qué han aprendido, plantear preguntas y aclarar las dudas que surgen (AP). La profesora intentará plantear cuestiones que den pie a preguntar y deba$\operatorname{tir}(\operatorname{ExP})$.

Una vez terminadas las exposiciones, la profesora resume en una presentación los contenidos teóricos y las cuestiones que han surgido a lo largo de la actividad (C) y, para concluir, se pide a los alumnos que valoren la actividad realizada, agradeciendo su participación (V).

Esta secuencia de actividades programadas aparece en la tabla 1 tal como fueron sucediéndose las sesiones, su finalidad y los contenidos programados. Los tiempos en todo momento han resultado muy justos, especialmente cuando participan los alumnos y la profesora. Tales intervenciones son muy enriquecedoras porque constituyen actividades de contraste. En ellas se ponen de manifiesto las necesidades de los alumnos y las explicaciones resultan muy provechosas para entender contenidos y superar obstáculos de aprendizaje.

Jornadas de Formación e Innovación Docente del Profesorado | № 2 (2019) Esta obra se distribuye con la licencia Creative Commons 
Tabla 1. Secuencia de actividades programada para el segundo ciclo de mejora

\begin{tabular}{|c|c|c|}
\hline $\begin{array}{l}\text { Descripción de la acti- } \\
\text { vidad (Tiempo) }\end{array}$ & Finalidad & Contenidos \\
\hline $\begin{array}{l}\text { Presentación del CIMA } \\
(30 \text { min) }\end{array}$ & $\begin{array}{l}\text { Explicación de las ac- } \\
\text { tividades, evaluación y } \\
\text { contenidos }\end{array}$ & \\
\hline Cuestionario (15 min) & \multicolumn{2}{|c|}{ Evaluar los conocimientos previos al CIMA } \\
\hline $\begin{array}{l}\text { Elección de tres ciu- } \\
\text { dades candidatas para } \\
\text { estudiar su clima (1,30 } \\
\text { min) }\end{array}$ & $\begin{array}{l}\text { Elección de una ciu- } \\
\text { dad por alumno para } \\
\text { representar los } 5 \text { gran- } \\
\text { des tipos y subtipos }\end{array}$ & $\begin{array}{l}\text { Zonas climáticas del } \\
\text { planeta } \\
\text { Clasificación de } \\
\text { Köppen }\end{array}$ \\
\hline $\begin{array}{l}\text { Descarga y trata- } \\
\text { miento de la informa- } \\
\text { ción }(1 \mathrm{~h})\end{array}$ & $\begin{array}{l}\text { Visor Global Climate } \\
\text { Monitor Tratamiento y } \\
\text { análisis de datos }\end{array}$ & $\begin{array}{l}\text { Obtención y organiza- } \\
\text { ción de la información } \\
\text { climática }\end{array}$ \\
\hline $\begin{array}{l}\text { Trabajo personal } \\
\text { Climograma }\end{array}$ & $\begin{array}{l}\text { Obtención de varia- } \\
\text { bles climáticas y re- } \\
\text { presentación gráfica }\end{array}$ & $\begin{array}{l}\text { Análisis estadístico } \\
\text { Diagramas climáticos }\end{array}$ \\
\hline $\begin{array}{l}\text { Trabajo en grupo } \\
\text { (3h,30 min) }\end{array}$ & $\begin{array}{l}\text { Comprender los facto- } \\
\text { res zonales }\end{array}$ & $\begin{array}{l}\text { Factores zonales de } \\
\text { los macroclimas del } \\
\text { planeta }\end{array}$ \\
\hline $\begin{array}{l}\text { Exposición de } \\
\text { resultados } \\
\text { (3h } 30 \mathrm{~min} \text { ) }\end{array}$ & $\begin{array}{l}\text { Presentación de los } \\
\text { resultados }\end{array}$ & $\begin{array}{l}\text { Factores explicativos } \\
\text { de los macroclimas y } \\
\text { climas regionales del } \\
\text { planeta }\end{array}$ \\
\hline Cuestionario (20 min) & \multicolumn{2}{|c|}{ Evaluar los conocimientos después del CIMA } \\
\hline $\begin{array}{l}\text { Resumen y valoración } \\
\text { (30 min) }\end{array}$ & $\begin{array}{l}\text { Resumen de conteni- } \\
\text { dos teóricos } \\
\text { Opinión de los estu- } \\
\text { diantes sobre el CIMA }\end{array}$ & $\begin{array}{l}\text { Factores zonales } \\
\text { Régimen de tempera- } \\
\text { turas y precipitaciones }\end{array}$ \\
\hline
\end{tabular}

En la valoración final los estudiantes pusieron de manifiesto la necesidad de disponer de más tiempo para las actividades. Cambiar el modelo de enseñanza-aprendizaje requiere también un espacio de adaptación a nuevas 
formas de funcionamiento, de trabajo y por el propio proceso continuo que supone la evaluación (Rivero y Porlán, 2017).

\section{Cuestionario inicial-final}

Los cuestionarios son un método muy eficaz para explorar las ideas y modelos previos de los alumnos. Sin ellos, u otras actividades similares, es dificil saber qué debemos mejorar y si realmente lo hemos conseguido tras la realización de las actividades. Por esta razón repetimos el mismo cuestionario al final como parte del proceso de evaluación. A causa de las limitaciones de tiempo debido a la distribución en bloques de la asignatura, el desarrollo del CIMA se inició antes de recibir toda la información y directrices en el Curso General de Docencia Universitaria (CGDU). Por esta razón, las preguntas del cuestionario han sido excesivamente académicas y no indagan en los conocimientos de los alumnos de forma desenfadada, como debían plantearse. El cuestionario se compone de las cinco preguntas siguientes:

1. ¿Conoces tipos diferentes de clasificaciones climáticas? Cita algún ejemplo

2. ¿Recuerdas los grandes tipos de clima que define Köppen?

3. En tu localidad ¿cómo se comportan las temperaturas en el año? ¿Sabes por qué?

4. ¿Llueve mucho dónde vives? ¿Qué ocurre a lo largo del año? ¿Sabes por qué?

5. ¿Hay alguna característica geográfica que influye sobre el clima de tu localidad?

Fue realizado inicialmente por los 15 estudiantes que habitualmente asisten a clase. Sin embargo, en la última sesión sólo asistieron a clase 11 estudiantes, por lo que 4 cuestionarios no pudieron ser utilizados para comparar los resultados de mejora tras las sesiones del CIMA. 


\section{Aplicación del ciclo de mejora en el aula}

Tras la presentación y explicación del CIMA, los alumnos muestran conformidad, aunque parecen desconcertados. Les doy por escrito la valoración de las actividades que contribuirán a la nota final. Realizan el cuestionario para evaluar los conocimientos previos y parecen bastante perdidos, lo que pone de manifiesto la necesidad de realizar actividades de contraste. La primera actividad consiste en pensar tres lugares del mundo que les gustaría estudiar, cada uno perteneciente a una de las tres bandas climáticas del planeta. En la siguiente sesión ponemos en común los tres lugares candidatos para estudiar su clima, entre los que deben elegir uno, para ir cubriendo entre todos los tipos climáticos. La clase resulta muy divertida nombrando lugares remotos, con nombres extraños y por conocer los intereses de los alumnos en su elección. Se crea un clima de confianza, complicidad y trabajo conjunto, por lo que hemos conseguido el primero de los principios que menciona Bain: crear un entorno para el aprendizaje crítico natural que favorezca el proceso de aprendizaje activo de los estudiantes (Bain, 2006). En este sentido considero que esta actividad ha salido muy bien, se han involucrado, nos hemos familiarizado con la clasificación de Köppen, con las características generales de sus grandes tipologías climáticas y hemos viajado por el mundo aprendiendo geografia. El resultado es un listado, confeccionado a medida que hacíamos la actividad, donde cada uno tiene un lugar con un tipo de clima diferente, pero que permite agruparlos en los 5 macroclimas (5 grupos).

La presentación del visor Global Climate Monitor, así como la descarga y el tratamiento de los datos parece interesarles. Esta actividad debería realizarse en un aula con ordenadores para que pudieran ir trabajando al mismo tiempo, pero el aula no dispone de ordenadores. Constato que este recurso tiene mucha potencialidad para la docencia de la climatología y resulta, además, de gran 
utilidad para otros campos de la geografía. A partir de entonces comienza el trabajo personal (actividades 2 y 3 ) con los datos hasta la realización del climograma (actividad 4) y la búsqueda de información sobre el clima del lugar elegido (actividad 5).

En la siguiente sesión comienza el trabajo en grupo (actividad 6); casi todos han elaborado su climograma, pero pocos traen manuales o información. No saben qué hacer ni cómo empezar y se encuentran un poco desconcertados. Ante esta dificultad tengo que ir grupo a grupo orientando, explicando cómo deben abordar la actividad. Constato nuevamente que los conocimientos más elementales ni se conocen, por lo que debería haber preparado instrucciones o haber desglosado en pasos los objetivos. Hay que pensar actividades más concretas, cortas, con un objetivo muy básico y sencillo ya que no saben trabajar desde un planteamiento más general. Además de este bloqueo, observo que no son capaces de describir la información que encuentran, copian párrafos de internet sin saber expresar ni redactar por sí mismos, o referir la información a su caso de estudio. No consiguen interpretar las características que observan en el climograma. Queda patente que los alumnos necesitan más ayuda y que hay que cambiar el modelo de enseñanza-aprendizaje. Sólo realizando este tipo de actividades de forma continuada pueden llegar a desarrollar estas habilidades básicas, aunque requeriría mucha constancia, así como replantear los contenidos de la asignatura.

La cuarta sesión se dedica exclusivamente al trabajo en equipos y desconcierta a los alumnos. Se nota que no están acostumbrados, les resulta un poco fastidioso aunque algunos se han motivado y se les ve trabajando muy bien. La clase dedicada a las presentaciones (actividad 7) pone de manifiesto que no han hecho lo suficiente y las exposiciones siguen la pauta tradicional de corta y pega de internet. En este sentido los resultados no han sido 
muy alentadores pero, precisamente por la necesidad de completar, siento que las explicaciones son más pertinentes y se dan en un contexto de participación conjunta, diferente a la clase magistral.

Para cerrar la actividad se presenta el mapa de contenidos y se explican de nuevo los objetivos, el sentido de la actividad que hemos realizado en el temario. Posiblemente sea mejor proporcionar a los alumnos este esquema inicialmente con una descripción y guía del CIMA. Recapitulamos contenidos conceptuales en el formato tradicional de presentación y realizan el cuestionario. En la valoración de la experiencia todos los alumnos están de acuerdo en que ha faltado tiempo, pero que han sido clases más amenas. Confiesan que no están acostumbrados a buscar información ya que generalmente se les proporciona. Sugieren que hubiese estado bien tener un ejemplo desarrollado como modelo a seguir para realizar la actividad propuesta, apreciación que me parece muy pertinente. Son buenas sugerencias a tener en cuenta la próxima vez.

\section{Evaluación del aprendizaje de los estudiantes}

A continuación, presentamos los resultados por preguntas describiendo los conocimientos previos que podían saber del curso anterior y que deseaba contrastar. Las tablas recogen los niveles de conocimiento que se establecen. Las escaleras de aprendizaje muestran el porcentaje de estudiantes en cada uno de ellos en el cuestionario inicial (izquierda) y final (derecha), así como los principales obstáculos identificados. Agruparemos las preguntas en dos bloques para presentar los resultados ya que sus contenidos están relacionados.

Los niveles de conocimiento en las preguntas 1 y 2 parten de los conocimientos adquiridos en el curso anterior sobre la clasificación climática de Köppen. Tal como 
recoge la tabla 2 la complejidad aumenta desde mencionar alguna clasificación climática, conocer el proceso de clasificación hasta saber que existen diferentes procedimientos para clasificar según sus objetivos. El nivel de conocimiento superior sería conocer los dos grandes tipos de clasificaciones climáticas, las descriptivas (basadas en los valores de las principales variables climáticas), y las genéticas (donde se incorporan los factores explicativos de los diferentes tipos de clima del mundo). Todos estos contenidos se encuentran en las presentaciones de los temas impartidos antes de comenzar el CIMA. En la tabla siguiente se presentan los niveles de conocimiento para la primera y segunda pregunta.

Tabla 2. Definición de los modelos de respuestas para las preguntas 1 y 2.

\begin{tabular}{|c|l|}
\hline NIVEL & PREGUNTA 1 \\
\hline E & No contesta, responde erróneamente o no sabe lo que es una clasificación climática o no cita ninguna \\
\hline D & Conoce el nombre de alguna clasificación climática \\
\hline C & Se acerca a poder definir que es una clasificación climática y cita algún ejemplo \\
\hline B & Puede definir qué es una clasificación climática y cita algún ejemplo \\
\hline A & Definición y tipos de clasificaciones climáticas con algún ejemplo \\
\hline \hline NIVEL & PREGUNTA 2 \\
\hline E & No contesta, responde erróneamente o no conoce los 5 tipos principales de la clasificación de Köppen \\
\hline D & Conoce el nombre de alguno de los 5 tipos principales \\
\hline C & Conoce los 5 tipos principales de Köppen y describe algo de ellos \\
\hline B & Conoce los 5 tipos principales de Köppen y sus rasgos principales (sabe el subtipo de su localidad) \\
\hline A & Conocey define los 5 tipos principales y subtipos de Köppen. Explica el clima de su localidad \\
\hline
\end{tabular}

A partir del cuestionario inicial y final se han elaborado las escaleras de aprendizaje. Mostramos la correspondiente a la primera pregunta en la figura 3. Podemos observar que inicialmente el mayor porcentaje de los alumnos se encuentra en los niveles más bajos, por lo es muy básico el conocimiento del que parten sobre las clasificaciones climáticas. 
Tras el CIMA, los porcentajes en los niveles más avanzados mejoran sustancialmente aunque en la pregunta 1 un 27,3 \% continúa en el nivel más bajo y ninguno llega al A. Pensamos que puede deberse al carácter teórico que no ha sido nuclear en el CIMA. Habría que reconsiderar cómo abordar estos contenidos. Los resultados de la pregunta 2 corroboran que los alumnos conocen la clasificación de Köppen y muchos de sus 5 grandes tipos. Es normal que no alcancen los escalones más altos porque implicaría recordar excesivos detalles, por eso los resultados mejoran al volver a manejar la clasificación. A pesar de ello, el incremento en el nivel A no es tan elevado como cabría esperar (9,1\%), lo que nos lleva a plantear la necesidad de nuevas actividades. Una de ellas podría ser aplicar los criterios de Köppen sobre los datos y verificar que realmente el clima de su localidad pertenece al tipo teórico.

Estas preguntas intentan indagar sobre los conocimientos previos que poseen sobre los factores zonales comentados en el mapa de contenidos (radiación solar y circulación atmosférica general) y azonales (relieve, cercanía al mar, etc.). Los niveles superiores implican poder explicar los mecanismos, las causas que determinan la existencia de diferencias en el comportamiento de la precipitación y la temperatura en los climas del planeta.

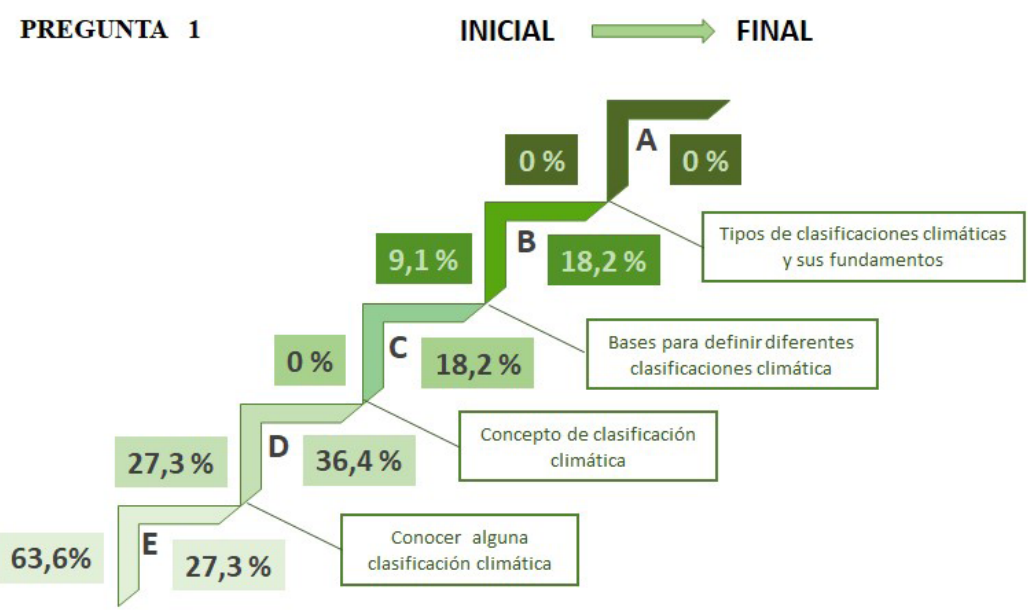

Figura 3: Escaleras de aprendizaje de las preguntas 1 y 2. Clasificaciones climáticas 
En la figura 3 se muestran los resultados obtenidos donde destacamos que, a pesar de ser buenos, sigue habiendo un porcentaje elevado en niveles bajos en alguna de las preguntas tras el CIMA, por lo que es necesario plantear nuevas actividades en relación a estos contenidos.

La pregunta uno es la que peores resultados ha obtenido por su carácter eminentemente teórico y por no ser imprescindible su contenido en la actividad, por lo que hay que plantear nuevas estrategias si se quieren impartir. En general, la mejoría en los resultados es notable tras la realización del CIMA, donde incluso un 50\% de la clase logra situarse en los niveles más altos para algunas cuestiones. En la valoración conjunta de las preguntas del cuestionario destacan los resultados de las preguntas 3 y 4 , que corresponden con las actividades que más han sido trabajadas en el CIMA.

Los resultados de las preguntas 3,4 y 5 se han agrupado ya que hacen alusión al conocimiento del clima de la localidad donde viven. La tabla 3 muestra los niveles en los modelos de repuesta sobre los factores zonales comentados en el mapa de contenidos (radiación solar y circulación atmosférica general) y azonales (relieve, cercanía al mar, etc.). Los niveles superiores implican poder explicar las causas que determinan la existencia de diferencias en el comportamiento de la precipitación y la temperatura en los climas del planeta. Los resultados mostrados en las escaleras se muestran en la figura 4.

Jornadas de Formación e Innovación Docente del Profesorado | № 2 (2019) Esta obra se distribuye con la licencia Creative Commons Reconocimiento-NoComercial-SinObraDerivada 
Tabla 3. Definición de los modelos de respuestas para las preguntas 3,4 y 5

\begin{tabular}{|c|c|}
\hline NIVEL & PREGUNTA3 \\
\hline $\mathrm{E}$ & No contesta, responde erróneamente o no dice nada sobre la radiación solar \\
\hline D & Conoce algo sobre radiación solar y su relación con el régimen térmico \\
\hline $\mathrm{C}$ & Conoce y explica algo sobre las diferencias latitudinales de la radiación solar y su relación con el régimen térmico \\
\hline B & Relaciona y explica la radiación solar con el régimen de temperaturas \\
\hline A & Conoce y explica los regimenes de temperatura en el planeta según las diferencias latitudinales de la radiación solar \\
\hline NIVEL & PREGUNTA 4 \\
\hline $\mathrm{E}$ & No contesta, responde erróneamente o no menciona ningún centro de acción que afectan a su localidad \\
\hline D & Conoce algún centro de acción que afectan a su localidad \\
\hline $\mathrm{C}$ & Conoce varios centros de acción que afectan a su localidad y dibuja algún esquema \\
\hline B & Conoce y explica varios centros de acción que afectan al régimen de lluvias de su localidad y dibuja algún esquema \\
\hline A & $\begin{array}{l}\text { Conoce y explica los centros de acción que afectan al régimen de lluvias de su localidad y díbuja el esquema en el } \\
\text { contexto del sistema climático mundial }\end{array}$ \\
\hline NIVEL & PREGUNTA5 \\
\hline $\mathrm{E}$ & No contesta, responde erróneamente ni nombra algún factor azonal que afecta al clima su localidad \\
\hline D & Conoce algún factor azonal que afecta al clima de su localidad \\
\hline C & Conoce algún factor azonal y sabe cómo afecta al clima de su localidad \\
\hline B & Conoce varios factores azonales y sabe cómo afectan al clima de su localidad \\
\hline A & Conoce los factores azonal es que afectan a su localidad. Explica los mecanismos y efectos sobre el clima \\
\hline
\end{tabular}

La dificultad principal en la actividad en grupo ha sido aplicar la teoría explicada en clase sobre los factores zonales a un tipo de macroclima concreto según Köppen. No consiguen comentar los climogramas que cada estudiante ha construido, ni relacionarlos con los factores que los determinan, por lo que habría que proporcionarles un modelo y desarrollar ejemplos con ellos durante el CIMA.

\section{Evaluación del CIMA puesto en práctica}

Los resultados globales de la aplicación del CIMA son muy positivos y demuestran la necesidad de cambiar el modelo de enseñanza-aprendizaje y los contenidos de la asignatura, ya que los modelos y conceptos previos de los alumnos sobre los que se basa el programa docente no responden a la realidad. El proceso de diseño y las

Jornadas de Formación e Innovación Docente del Profesorado | № 2 (2019) Esta obra se distribuye con la licencia Creative Commons Reconocimiento-NoComercial-SinObraDerivada Internacional (CC BY-NC-ND 4.0.) 
herramientas aprendidas (mapa de contenidos, escaleras, etc.) han resultado muy útiles, abren nuevos retos docentes y han supuesto una renovación en mi motivación académica. En relación al ambiente en el aula y con los alumnos han sido también muy satisfactoria, las clases han sido más amenas y el CIMA ha conseguido quitar a la profesora del centro. Se ha puesto de manifiesto que la pregunta central es excesivamente general para los alumnos, hay que plantear una secuencia de actividades más sencillas y concretas. Hay que revisar la secuencia de actividades, ajustándola a las indicaciones de Finkel: generar una secuencia de preguntas que se construyen sobre la pregunta original, más centradas y directas y que deben necesariamente darse por escrito a los estudiantes (Finkel, 2008).

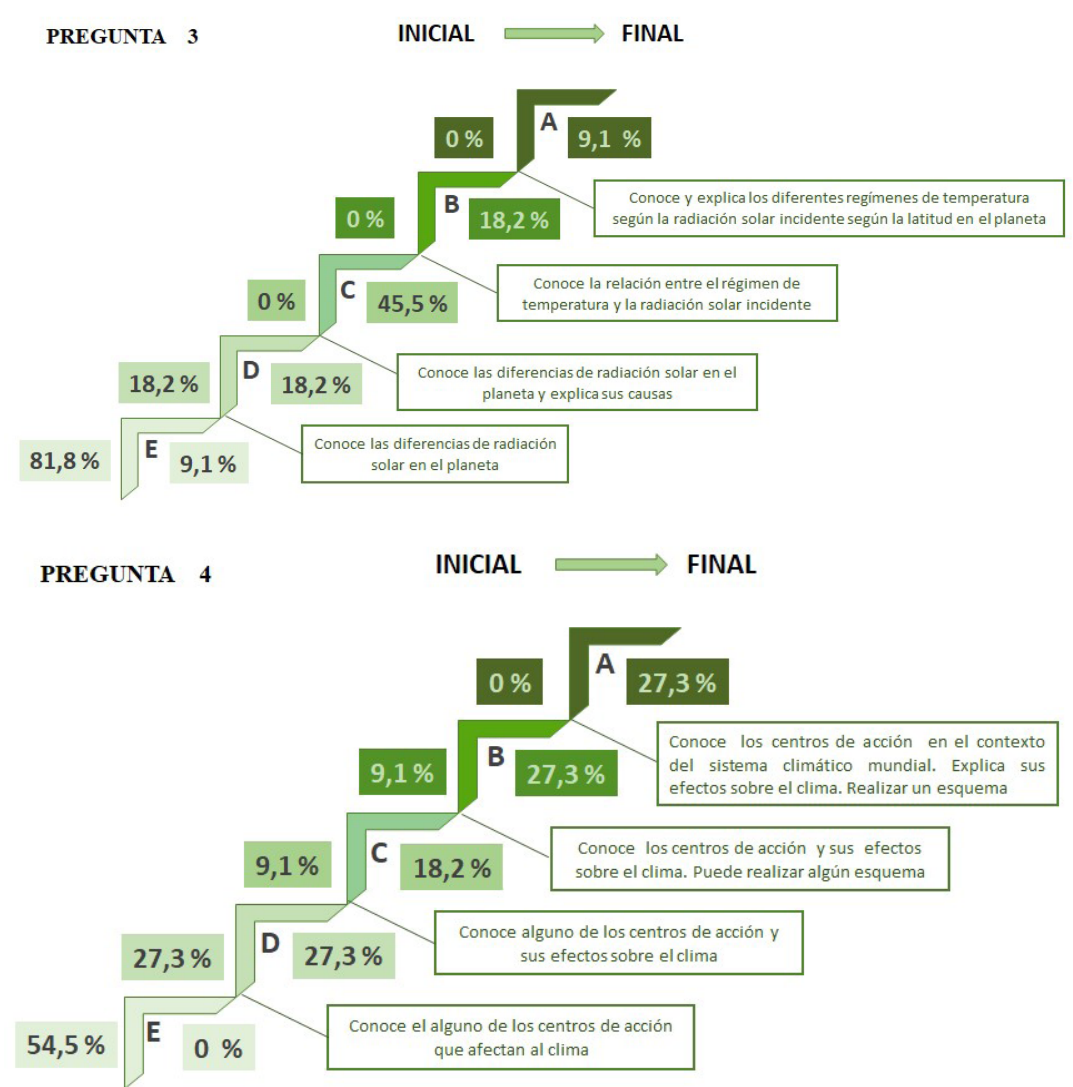

Jornadas de Formación e Innovación Docente del Profesorado | № 2 (2019) Esta obra se distribuye con la licencia Creative Commons Reconocimiento-NoComercial-SinObraDerivada 4.0 Internacional (CC BY-NC-ND 4.0.) 
INICIAL FINAL

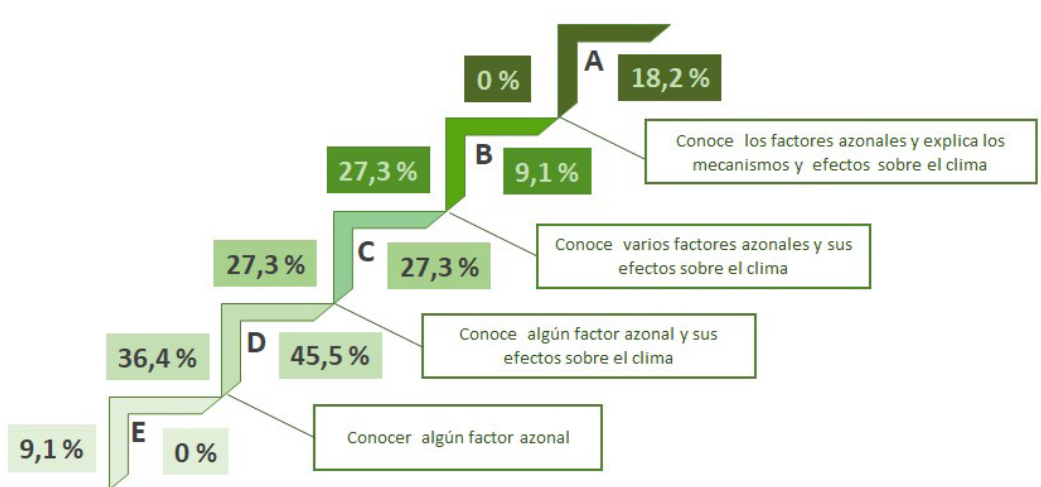

Figura 4: Escalera de aprendizaje de las preguntas 3, 4 y 5. El clima de tu localidad

Hay que diseñar nuevas actividades destinadas a superar dificultades en el aprendizaje, relacionadas con los siguientes aspectos:

- Trabajar las competencias relacionadas con el comentario, descripción y explicación de los mecanismos que subyacen en lo que observamos. En definitiva, poder hablar de los contenidos teóricos partiendo de casos reales.

- Superar las carencias y errores en los conocimientos básicos de climatología.

- Conseguir que trabajen los materiales de clase.

- Realizar más actividades con el visor Global Climate Monitor.

- Motivar la búsqueda de información contrastada.

- Mejorar las habilidades en la exposición de trabajos en clase.

- Identificar contenidos conceptuales fuera del formato de clase magistral.

- Mejorar el nivel de motivación y rigor.

La aplicación del CIMA evidencia la necesidad de dedicar más tiempo a las actividades programadas y al proceso 
de evaluación, ya que alumnos y profesores deben trabajar bajo un nuevo contexto de enseñanza-aprendizaje.

Sin duda, el reto futuro se centra en incorporar actividades relacionadas con los contenidos actitudinales, que no han podido ser abordados en el CIMA. Trabajar en un contexto de cambio climático global plantea un sinfin de cuestiones, se trata del mayor problema ambiental al que se enfrenta la humanidad y que debe llegar a las aulas. 


\section{Referencias bibliográficas}

Bain, K. (2006). Lo que hacen los mejores profesores universitarios. Valencia: Universitat de Valencia.

Camarillo-Naranjo, J. M., Álvarez-Francoso, J. I., Limones-Rodríguez, N., Pita-López, M. F. y Aguilar-Alba, M. (2018). The global climate monitor system: from climate data-handling to knowledge dissemination. International Journal of Digital Earth, 1-21. doi:10.1080/17538947.2018 .1429502

Edwards, P. N., Mayernik, M. S., Batcheller, A. L., Bowker, G. C., \& Borgman, C. L. (2011). Science friction: Data, metadata, and collaboration. Social Studies of Science, 41(5), 667-690. doi:10.1177/0306312711413314

Finkel, D. (2008). Dar clase con la boca cerrada. Valencia: Universitat de València.

Overpeck, J. T., Meehl, G. A., Bony, S., \& Easterling, D. R. (2011). Climate data challenges in the 21st century. Science, 331(6018), 700-702. dol:10.1126/science.1197869

Petre, A. L., Ramos Calcedo, G., Perdigón Melón, J. A., Cabos Narváez, W., García-Abad Alonso, J. J., Guardiola Soler, J., \& Carbajo Elena, J. B. (2014). La utilización del portafolio discente interdisciplinar como metodología en la evaluación de la asignatura de meteorología, climatología y contaminación atmosférica de grado en ciencias ambientales. Tendencias pedagógicas, 23, 7796. Recuperado de https://dialnet.unirioja.es/servlet/ articulo?codigo $=4661029$

Rivero, A. y Porlán, R. (2017). La evaluación en la enseñanza universitaria. En R. Porlán (Coord.), Enseñanza universitaria. Como mejorarla (pp. 73-91). Madrid: Morata.

Rodríguez Puebla, C. (2010). Diseño de experiencias para el estudio de fenómenos climáticos. Memoria ID-0206. Ayudas de la Universidad de Salamanca para la innovación docente, curso 2009-2010. Recuperado de http:// hdl.handle.net/10366/81786 